\title{
THE EFFECT OF Fe SUBSTITUTION ON THE CHARGE DENSITY WAVE IN VSe
}

\author{
F. J. DI SALVO and J. V. WASZCZAK \\ Bell Laboratories, Murray Hill, New Jersey 07974, U. S. A.
}

\begin{abstract}
Résumé. - Les effets de la substitution de fer dans le composé $\mathrm{V}_{1-x} \mathrm{Fe}_{x} \mathrm{Se}_{2}$ sont comparés à ceux mesurés dans le composé 1T-Ta ${ }_{1-x} \mathrm{Fe}_{x} \mathrm{Se}_{2}$. Dans le composé pur $\mathrm{VSe}_{2}$ une onde de densité de charge $(\mathrm{CDW})$ apparaît au-dessous de $T_{0}=112 \mathrm{~K}$, à la différence du composé 1T-TaSe ${ }_{2}$ où $T_{0} \approx 600 \mathrm{~K}$. Plusieurs phénomènes associés à la présence du fer substitutionnel sont observés dans le composé $1 \mathrm{~T}-\mathrm{Ta}_{1-x} \mathrm{Fe}_{x} \mathrm{Se}_{2}$ :
\end{abstract}

(1) la $\mathrm{CDW}$ est stable à $300 \mathrm{~K}$ pour $x \lesssim 0,15$,

(2) le niveau commensurable de la CDW est supprimé à $x \approx 0,06$,

(3) la conductivité décroît rapidement vers zéro à basses températures quand $x \geqslant x_{\mathrm{c}} \simeq 0,15$ (localisation d'Anderson),

(4) le fer possède une transition d'un état bas spin à un état de haut spin pour des températures croissantes. On trouve que pour le fer substitutionnel dans le VSe $\mathrm{Ve}_{2}$ : (1) la CDW est éliminée pour $x \gtrsim 0,035$, (2) le fer est non magnétique (bas spin), (3) jusqu'à la plus haute concentration étudiée $(0 \leqslant x \leqslant 0,2)$, on ne peut mettre en évidence la localisation d'Anderson. Les résultats sont cohérents avec une CDW beaucoup moins stable dans $\mathrm{VSe}_{2}$ que dans le 1T-TaSe ${ }_{2}$.

\footnotetext{
Abstract. - We compare the effect of $\mathrm{Fe}$ substitution in $\mathrm{V}_{\mathrm{1}-x} \mathrm{Fe}_{2} \mathrm{Se}_{2}$ with the previously measured properties of $1 \mathrm{~T}-\mathrm{Ta}_{1-x} \mathrm{Fe}_{x} \mathrm{Se}_{2}$. In pure $\mathrm{VSe}_{2}$ a Charge Density Wave (CDW) appears below $T_{0}=112 \mathrm{~K}$, while the CDW in $1 \mathrm{~T}-\mathrm{TaSe}_{2}$ occurs below $T_{0} \approx 600 \mathrm{~K}$. Several phenomena associated with $\mathrm{Fe}$ substitution are observed in $1 \mathrm{~T}-\mathrm{Ta}_{1-x} \mathrm{Fe}_{x} \mathrm{Se}_{2}$ :

(1) the CDW is stable at $300 \mathrm{~K}$ for $x$ up to at least 0.15 ,

(2) the commensurate $\mathrm{CDW}$ state is suppressed at $x \approx 0.06$,

(3) the conductivity decreases rapidly to zero at low temperatures when $x \gtrsim x_{\mathrm{c}} \simeq 0.15$ (Anderson localization),

(4) the Fe shows a low spin to high spin transition as the temperature is increased. We find for $1 \mathrm{~T}-\mathrm{V}_{1-x} \mathrm{Fe}_{x} \mathrm{Se}_{2} x \leqslant$; (1) the CDW is eliminated for $x \gtrsim 0.035$, (2) the Fe is nonmagnetic (low spin), (3) to the concentrations studied $(x=0.2)$, no evidence for Anderson localization. The results are consistent with the CDW being much less stable in $\mathrm{VSe}_{2}$ than in $1 \mathrm{~T}-\mathrm{TaSe}_{2}$.
}

1. Introduction. - We present recent studies of the effects of cation substitution on the Charge Density Wave (CDW) transition in $\mathrm{VSe}_{2}$. These results are compared to those obtained in $\mathrm{TaSe}_{2}[1,2,3]$ and to the ideas expressed in phenomenological Landau theories $[2,4]$. In order to make an orderly comparison, some of our previous results will be first reviewed.

A Charge Density Wave is a periodic static distortion of the lattice and conduction electron density that occurs in most of the metallic layered compounds below an onset temperature $T_{0}$. The wave vector of this distortion, $q$, is determined by the Fermi surface and is generally incommensurate with the lattice. Frequently, the $q$ changes slightly to become commensurate with the lattice in a first order transition at $T_{\mathrm{d}}<T_{0}$.

Table I lists some of the CDW unstable layered compounds with the onset temperature $T_{0}$, lock in temperature $T_{\mathrm{d}}$, and approximate $\mathrm{CDW}$ wavelength $(\lambda=2 \pi / \mathrm{q})$. $\mathrm{VSe}_{2}$ has the largest $\lambda$ and the lowest $T_{0}$ of the 1T structure compounds. Here we will compare

\section{TABLE I}

We list the $\mathrm{CDW}$ onset temperature, $T_{0}$, the temperature of a discontinuous transition to the commensurate $\mathrm{CDW}$ state, $T_{\mathrm{d}}$, and the approximate wavelength of the $\mathrm{CDW}$ in the incommensurate state

\begin{tabular}{|c|c|c|c|c|}
\hline Material & $T_{0}(\mathrm{~K})$ & $T_{\mathrm{d}}(\mathrm{K})$ & $\lambda \mathrm{oDw}$ & Reference \\
\hline $1 \mathrm{~T}-\mathrm{TaSe}_{2}$ & $\approx 600 \mathrm{~K}$ & $473 \mathrm{~K}$ & $\approx 3.5 a$ & 1,3 \\
\hline $2 \mathrm{H}-\mathrm{TaSe}_{2}$ & $122 \mathrm{~K}$ & $\approx 95 \mathrm{~K}$ & $\approx 3.0 \mathrm{a}$ & 1,2 \\
\hline $2 \mathrm{H}-\mathrm{NbSe}_{2}$ & $32 \mathrm{~K}$ & - & $\approx 3.0 \mathrm{a}$ & 2 \\
\hline $1 \mathrm{~T}-\mathrm{VSe}_{2}$ & $112 \mathrm{~K}$ & $\approx 80 \mathrm{~K}$ & $\approx 4.0 \mathrm{a}$ & 5,6 \\
\hline $1 \mathrm{~T}-\mathrm{TiSe}_{2}$ & 202 & & $2 a$ & 5,7 \\
\hline
\end{tabular}

the properties of $\mathrm{VSe}_{2}$ to those of 1T-TaSe $\mathrm{Tince}_{2}$ the structures are the same, and to $2 \mathrm{H}-\mathrm{TaSe}_{2}$ since the physical properties are quite similar to those of $\mathrm{VSe}_{2}$ [5]. We find that the CDW state of $\mathrm{VSe}_{2}$ is very sensitive to cation disorder, the $\mathrm{CDW}$ state is eliminated when approximately $3 \% \mathrm{Fe}$ is substituted for $\mathrm{V}$. 
2. Effects of doping in 1T-TaSe susceptibility $(\chi)$ of $1 \mathrm{~T}-\mathrm{TaSe}_{2}$ and $2 \mathrm{H}-\mathrm{TaSe}_{2}$ is given in figure 1 and the electrical resistivity $(\rho)$ in figure 2. The wide difference in the properties of

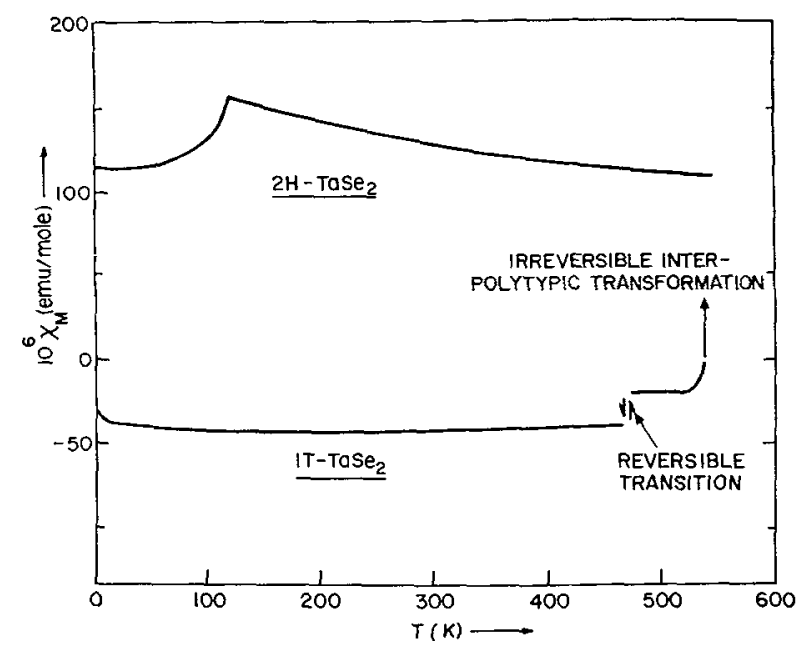

FIG. 1. - The magnetic susceptibility of $2 \mathrm{H}-\mathrm{TaSe}_{2}$ powder shows a sudden decrease below $T_{0}$ but remains paramagnetic, while $1 \mathrm{~T}-\mathrm{TaSe}_{2}$ is diamagnetic even above the first order transition at $T_{\mathrm{d}}$

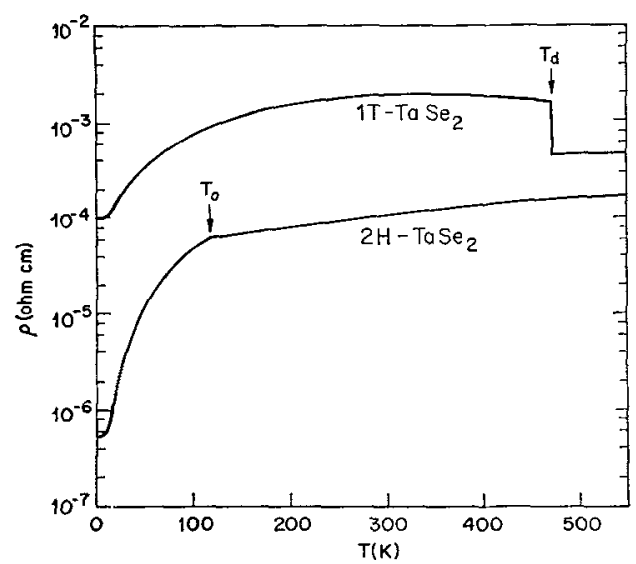

FIG. 2. - The electrical resistivity of $2 \mathrm{H}-\mathrm{TaSe}_{2}$ parallel to the layers shows a small increase below $T_{0}$ then a rapid drop. The electrical resistivity of $1 \mathrm{~T}-\mathrm{TaSe}_{2}$ shows a sharp increase at $T_{\mathrm{d}}$, below which $\rho$ is more than an order of magnitude above that of the $2 \mathrm{H}$ polymorph.

these polymorphs is due in part to their different band structure [8] and to the possible different mechanisms for CDW formation [9]. Here we note the primary differences :

1) $\chi$ of $2 \mathrm{H}-\mathrm{TaSe}_{2}$ shows strong Pauli paramagnetism both above and below $T_{0}=122 \mathrm{~K}$, while $1 \mathrm{~T}-\mathrm{TaSe}_{2}$ is diamagnetic below $T_{0} \approx 600 \mathrm{~K}$.

2) The resistivity of $2 \mathrm{H}-\mathrm{TaSe}_{2}$ drops rapidly below $T_{0}=122 \mathrm{~K}$, while the resistivity of $1 \mathrm{~T}-\mathrm{TaSe}_{2}$ is larger than that of $2 \mathrm{H}-\mathrm{TaSe}_{2}$ even at high temperatures $(\sim 500 \mathrm{~K})$.

The difference below $T_{0}$ are also related to the fraction of Fermi surface destroyed by the CDW induced gaps. In $1 \mathrm{~T}-\mathrm{TaSe}_{2}$ approximately $90 \%$ of the Fermi surface is lost, while in $2 \mathrm{H}-\mathrm{TaSe}_{2} 15 \%$ or less is lost $[3,10]$.

The effects of cation substitution have been studied in $1 \mathrm{~T}-\mathrm{TaSe}_{2}$, since in most cases even very small substitution appears to make the $2 \mathrm{H}$ polymorph unstable [3]. Cation substitution has been used to show that decreasing conduction electron concentration decreases the CDW wavelength, for example in $1 \mathrm{~T}-\mathrm{Ta}_{1-x} \mathrm{Ti}_{x} \mathrm{Se}_{2}[1,3]$. Cation substitution also produces disorder. The effects of disorder in $1 \mathrm{~T}-\mathrm{TaSe}_{2}$ are threefold :

1) The onset temperature is slowly reduced.

2) The lock in temperature is rapidly reduced. The commensurate state does not occur beyond a critical doping level, as illustrated in figure 3 . The effects of doping on $T_{0}$ and $T_{\mathrm{d}}$ agree at least qualitatively with the Landau model proposed by McMillan [4].

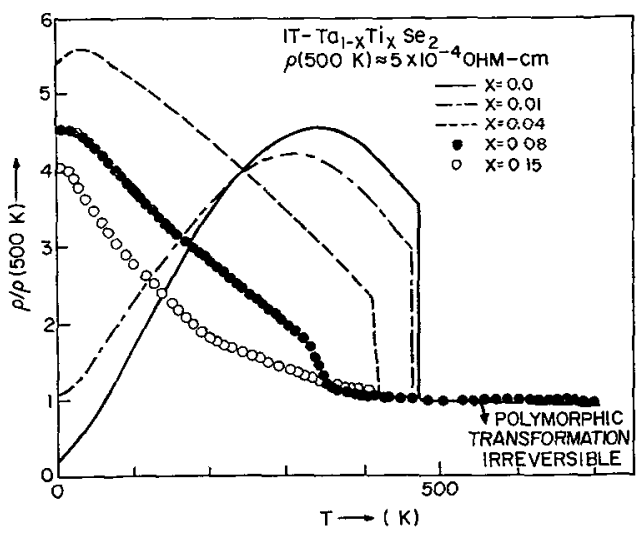

Frg. 3. - In 1T-Ta $\mathrm{Ta}_{1-x} \mathrm{Ti}_{x} \mathrm{Se}_{2}$ cation doping reduces $T_{a}$ rapidly so that the commensurate state does not occur for $x \gtrsim 0.1$. The low temperature resistivity remains high ane to enhanced impurity scattering.

3) The low temperature resistivity is greatly increased over that of the pure material (see Fig. 3). For some dopants, the resistivity is infinite at zero temperature when the doping is beyond a certain minimum level [11]. For example in $1 \mathrm{~T}-\mathrm{Ta}_{1-x} \mathrm{Fe}_{x} \mathrm{Se}_{2}$ the resistivity for $x \gtrsim 0.15$ diverges at low temperatures as shown in figure 4 .

This last case of $\mathrm{Fe}$ doping is particularly interesting. Mössbauer data show that the $\mathrm{Fe}$ is divalent in 1T- $\mathrm{Ta}_{1-x} \mathrm{Fe}_{x} \mathrm{Se}_{2}$ [12]. $2 x$ of the Ta atoms become pentavalent for overall charge compensation, reducing the conduction electron density to $1-3 x$ of that in $1 \mathrm{~T}-\mathrm{TaSe}_{2}$. Consequently, semiconducting like properties at $x=\frac{1}{3}$ are not unexpected. However when $0.15<x<\frac{1}{3}$, the loss of electron mobility at low temperatures is due to the interaction of increasing randomness and decreasing occupied bandwidth. Within simple models, the localization occurs when the amplitude of the random part of the potential 


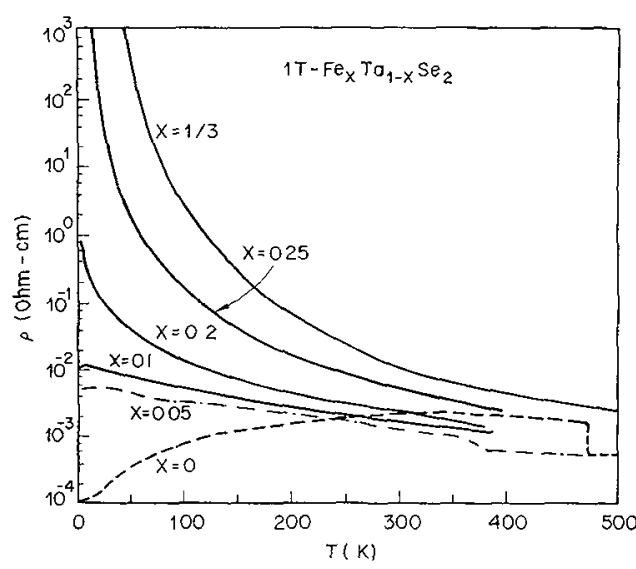

FIG. 4. - The random potential of some dopants, such as $\mathrm{Fe}$ in $1 \mathrm{~T}-\mathrm{Ta}_{1-x} \mathrm{Fe}_{x} \mathrm{Se}_{2}$, produce Anderson localization of the remaining carriers at low $T$ when $x>x_{c}$.

is equal to the bandwidth. Landau theories predict that the impurity potential close to a CDW instability will be strong and long range [4], rather than being screened out in a short Thomas Fermi length, as in normal metals. The CDW enhances the random potential over what is obtained in normal metals, producing Anderson localization [13] when the concentration of $\mathrm{Fe}, \mathrm{Co}$, or $\mathrm{Ni}$ is above a critical amount in $1 \mathrm{~T}-\mathrm{TaSe}_{2}$ [11].

Finally we note that $1 \mathrm{~T}-\mathrm{Ta}_{1-x} \mathrm{Fe}_{x} \mathrm{~S}_{2}$ (or $\mathrm{Se}_{2}$ ) exhibits a low spin to high spin transition of the $\mathrm{Fe}^{2+}$ with increasing temperature [12], as observed for example in the magnetic susceptibility of $1 \mathrm{~T}-\mathrm{Ta}_{0.9} \mathrm{Fe}_{0.1} \mathrm{Se}_{2}$ (Fig. 5). This transition occurs

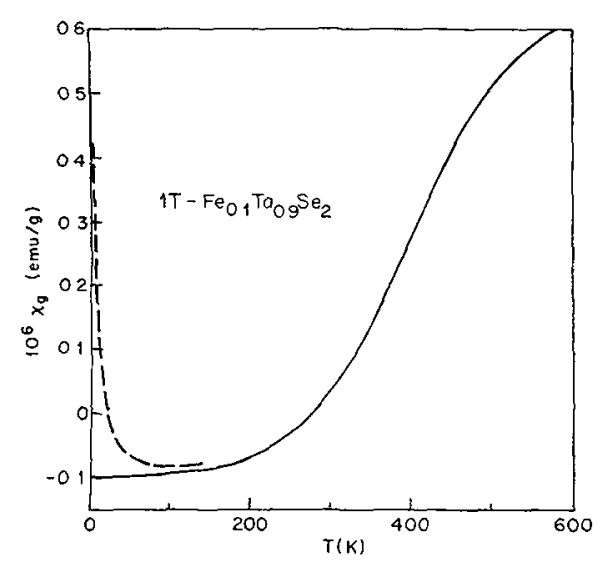

FIG. 5. - In the case of Fe doping in 1T-TaSe 2 a low spin to high spin transition of $\mathrm{Fe}^{2+}$ is observed with increasing temperature. The dashed curve indicates a small Curie tail, due to impurities or a small fraction of intercalated $\mathrm{Fe}$, that has been subtracted from the data.

when the crystal field $(10 D q)$ and Hund's rule energy $\left(E_{H}\right)$ are close to equal. The crystal field is determined primarily by the near neighbour Se positions and distances from the $\mathrm{Fe}$, and the latter parameters are fixed by the parent $1 \mathrm{~T}-\mathrm{TaSe}_{2}$ lattice. From the measurements we know that $\Delta=10 \mathrm{Dq}-E_{\mathrm{H}}$ is small at high temperatures $\left(T \gtrsim T_{0}\right)$. However, $10 D q$ is expected to have an unusual temperature dependence due to the large amplitude of the CDW $\left(\approx\right.$ one electron/atom) attained well below $T_{0}[14,15]$ We find that $\Delta$ in fact increases at low temperatures, rapidly cutting off the high spin state.

In the case of the $1 \mathrm{~T}$ polytypes it is evident that not only does cation doping effect the CDW but also the CDW effects the magnetic behaviour and the scattering potential of the dopants. Since the variety of phenomena observed is so large in the case of Fe doping in $1 \mathrm{~T}-\mathrm{TaSe}_{2}$, we have begun a comparative study of $1 \mathrm{~T}-\mathrm{V}_{1-x} \mathrm{Fe}_{x} \mathrm{Se}_{2}$.

3. Sample preparation. - Powder samples were prepared from the elements by reaction at $650^{\circ} \mathrm{C}$ in a small excess of Se. The samples were homogenized by grinding the reacted powder and pressing into pellets with subsequent reheating at $650^{\circ} \mathrm{C}$ (twice). At higher reaction temperatures $\mathrm{V}_{1+x} \mathrm{Se}_{2}$ forms with $x>0$ even when prepared in excess Se. Single crystals were grown by iodine vapor transport from these powders, again in excess $\mathrm{Se}$, at a growth temperature of $650^{\circ} \mathrm{C}$.

The $1 \mathrm{~T}$ structure is retained for $\mathrm{Fe}$ substitution up to $x=\frac{1}{2}\left(\mathrm{~V}_{1 / 2} \mathrm{Fe}_{1 / 2} \mathrm{Se}_{2}\right)$. No extra lines are observed in powder X-ray diffraction, indicating that the $\mathrm{Fe}$ substitution is random, as in $1 \mathrm{~T}-\mathrm{Ta}_{1-x} \mathrm{Fe}_{x} \mathrm{Se}_{2}$ [3] Magnetic susceptibility indicates that $\mathrm{Fe}$ is divalent and low spin (see later). At $x=\frac{1}{3}$ we would expect all the $\mathrm{V}$ to be pentavalent, and further substitution impossible. However, in $\mathrm{VSe}_{2}$ the $\mathrm{V}$ d-band and $\mathrm{Se}$ valence band overlap, as shown by photoemission: [16] So that as the d-band is emptied by production of $\mathrm{V}^{5+}$, holes are produced in the valence band. At $x=\frac{1}{2}$ there should be 0.5 holes per Se atom.

4. Results. - The magnetic susceptibility and resistivity of pure $\mathrm{VSe}_{2}$ are shown in figure 6 . An anomaly

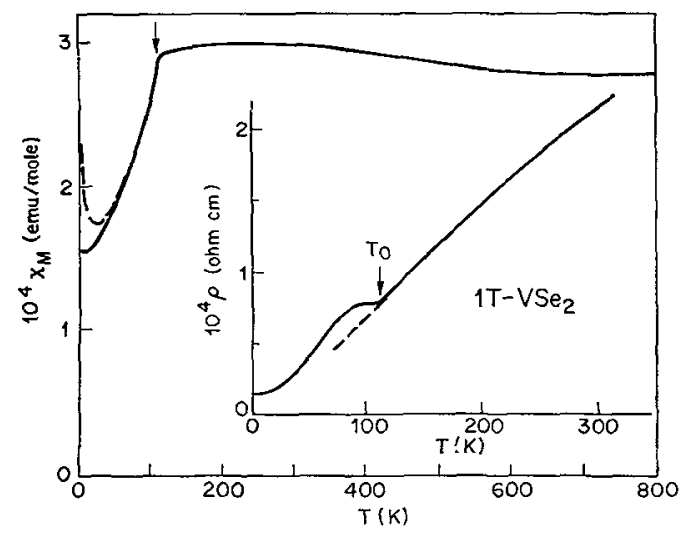

FIG. 6. - The magnetic susceptibility of $1 \mathrm{~T}-\mathrm{VSe}_{2}$ powder shows a rapid drop below $T_{0}=112 \mathrm{~K}$. Again a small Curie tail, indicated by the dashed curve, has been subtracted out. The inset shows the anomaly apparent below $T_{0}$ in the resistivity (parallel to the layers). 
is apparent in both properties at the CDW onset temperature, $T_{0}=112 \mathrm{~K}$. While $V S_{2}$ has a $1 \mathrm{~T}$ structure, the CDW anomalies have some similarity to those of $2 \mathrm{H}-\mathrm{TaSe}_{2}$ (see Figs. 1 and 2) [17]. At $T_{\mathrm{d}}$ the anomalies in these properties are too weak to be seen on this scale [5]. From the decrease in $\chi$ below $T_{0}$ and the increase in $\rho$, we estimate that approximately $30 \%$ of the Fermi surface is lost in the CDW transition. This latter fact, along with the lower $T_{0}$, suggests that the CDW has a smaller amplitude in $\mathrm{VSe}_{2}$ than in $1 \mathrm{~T}-\mathrm{TaSe}_{2}$.

When $F e$ is substituted for $V$ the CDW onset temperature and amplitude are rapidly suppressed, as determined by the magnetic susceptibility (Fig. 7).

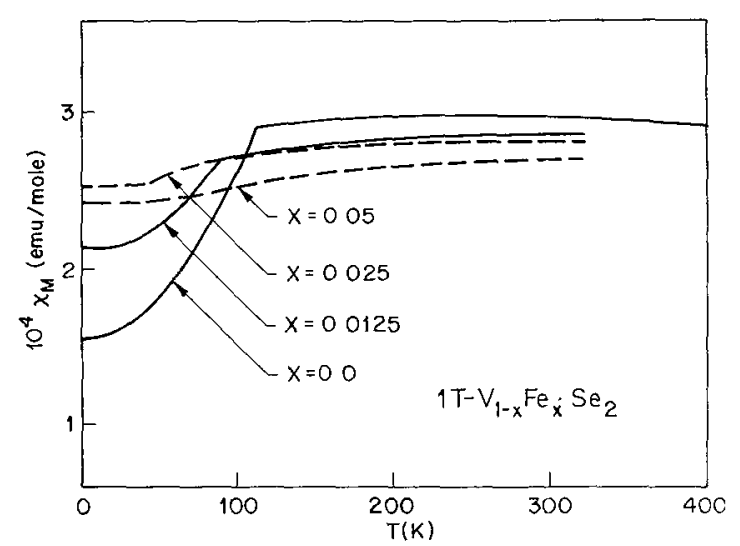

FIG. 7. - The magnetic susceptibility of $\mathrm{V}_{1-x} \mathrm{Fe}_{x} \mathrm{Se}_{2}$ powders clearly shows the suppression of $T_{0}$ and $\Delta \chi$, the decrease in $\chi$ below $T_{0} . \quad T_{0}=0$ at $x \approx 0.035$. Small Curie contributions, comparable to that shown in figure 6 , were subtracted from the data.

We estimate that $T_{0}=0$ at $x=0.035$. The smooth but small decrease in $\chi$ observed when $x>0.035$ is probably related to the decrease in the density of states at the Fermi level induced by local distortions about the $\mathrm{V}$ sites. These distortions will occur even above $T_{0}$, or, as in the case here, when the CDW is incipient (not quite stable) [4].

The suppression of $T_{0}$ is almost as rapid when $\mathrm{Ti}$ is substituted for $\mathrm{V}$ as shown in figure 8 . Ti valence is $4+$ and does not produce $\mathrm{V}^{5+}$ for charge compensation as does $\mathrm{Fe}$. But even for this weak perturbation of the order in $\mathrm{VSe}_{2}, T_{0}$ is reduced to zero at $x$ approximately 0.05 .

At large $x$, the low temperature susceptibility increases rapidly and is given by $\chi(T)=\chi_{0}+C / T$, where $\chi_{0}$ is a constant (see Fig. 9). The Curie constant, $C$, gives a $\mu_{\text {eff }}$ of $0.6 \mu_{\mathrm{B}}$ and $0.7 \mu_{\mathrm{B}}$ per Fe at $x=\frac{1}{3}$ and $\frac{1}{2}$ respectively. This moment could arise in several ways :

1) each Fe has the low moment calculated;

2) a small amount of Fe or $V$ is in the Van der Waals region between the layers. In this case either would be magnetic $\left(\mathrm{Fe}^{2+} S=2\right.$, or $\mathrm{V}^{3+} S=1$ ) [18].

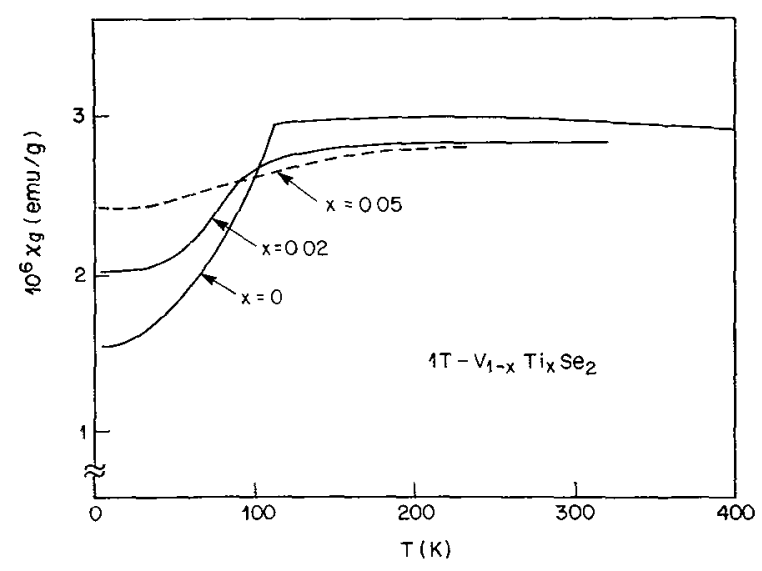

FIG. 8. - The magnetic susceptibility of $\mathrm{V}_{1-x} \mathrm{Ti}_{x} \mathrm{Se}_{2}$ shows that $T_{0}$ is not initially suppressed as fast as with $\mathrm{Fe}$ doping, but $T_{0}$ is reduced to zero near $x=0.05$.

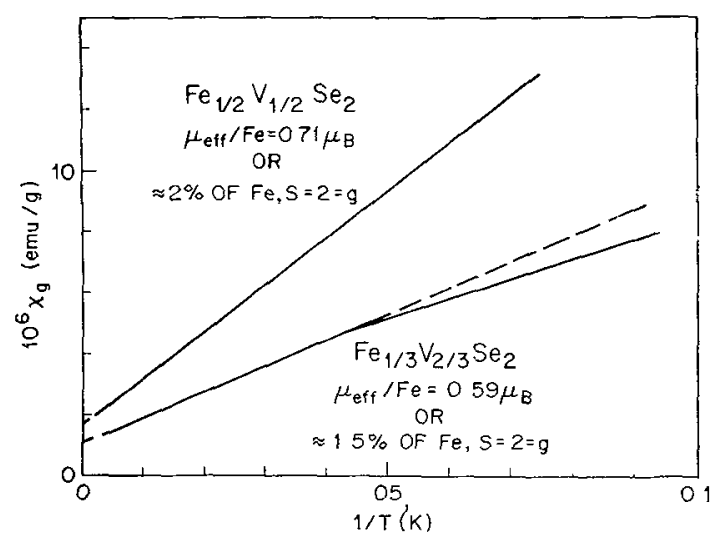

FIG. 9. - The magnetic susceptibility of $\mathrm{V}_{2 / 3} \mathrm{Fe}_{1 / 3} \mathrm{Se}_{2}$ and $\mathrm{V}_{1 / 2} \mathrm{Fe}_{1 / 2} \mathrm{Se}_{2}$ plotted vs. $1 / T$ shows a much larger Curie tail at low temperatures than when $x \leqslant 0.10$. However, this tail is probably due to a few percent of intercalated Fe or V.

The latter possibility [2] seems most likely, since even stoichiometric $\mathrm{VSe}_{2}$ itself is difficult to prepare. The paramagnetic $\chi_{0}$ obtained for these two samples may arise from two sources : the Pauli paramagnetism of the expected Se holes and the van Vleck paramagnetism of the $\mathrm{Fe}^{2+}$ ion.

There is no evidence at any $\mathrm{Fe}$ concentration of a low spin to high spin transition of the Fe with increasing temperature. This is not surprising for two reasons :

(1) The Fe-Se bond length imposed by the parent $\mathrm{VSe}_{2}$ lattice is shorter than in $1 \mathrm{~T}-\mathrm{TaSe}_{2}$. Thus $10 \mathrm{Dq}$ will be larger.

(2) The modulation of $10 D q$ by the $\mathrm{CDW}$ will be weaker in $\mathrm{VSe}_{2}$ than in $1 \mathrm{~T}-\mathrm{TaSe}_{2}$.

The electrical resistivity of $1 \mathrm{~T}-\mathrm{V}_{1-x} \mathrm{Fe}_{x} \mathrm{Se}_{2}$ crystals is shown figure 10 for current parallel to the layers. The anomaly signifying the $\mathrm{CDW}$ onset is rapidly lost as expected. By $x \gtrsim 0.1$ the impurity scattering is quite large, even though the CDW is fully suppressed 


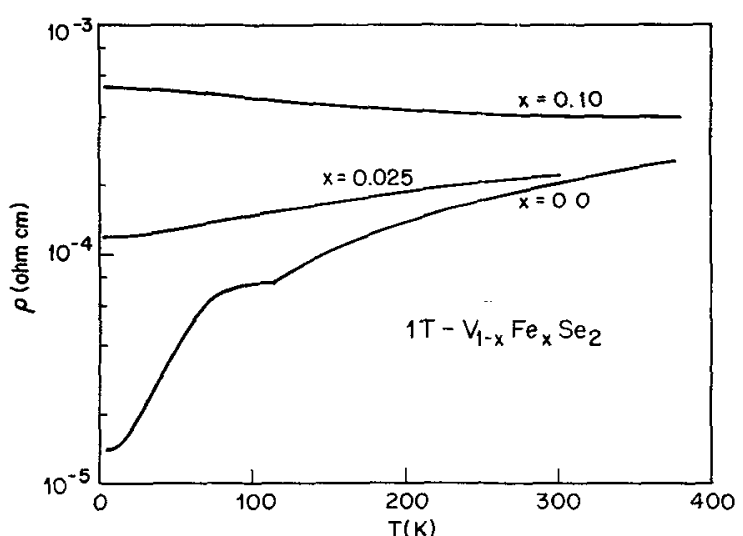

FIg. 10. - The electrical resistivity of $\mathrm{V}_{1_{x}} \mathrm{Fe}_{x} \mathrm{Se}_{2}$ parallel to the layers also shows that $T_{0}$ is rapidly suppressed, the anomaly characterizing the CDW onset is lost. Impurity scattering at low temperatures is likely enhanced by local CDW distortions.

At $x=0.2$ the resistivity is similar to that shown for $x=0.1$. Again this is due to the local distortions about the impurities that are expected to occur when the material is close to a CDW instability [19].

At present we have not yet been able to prepare single crystals of $\mathrm{V}_{1-x} \mathrm{Fe}_{x} \mathrm{Se}_{2}$ for $x \geqslant \frac{1}{3}$ that are large enough for resistivity measurements. Consequently we can not say for certain that Anderson localization will occur. However, we feel that the properties already measured suggest that such a localization will not occur. There are two reasons for this opinion :

(1) The carrier concentration most likely does not get much below one per $\mathrm{V}$ atom. (Remember, even at $x=\frac{1}{2}$ there will be 0.5 holes per Se or 1 per V.)
Consequently, the bandwidth remains relatively large even with considerable $\mathrm{Fe}$ doping.

(2) The CDW amplitude is smaller in $\mathrm{VSe}_{2}$ than in $1 \mathrm{~T}-\mathrm{TaSe}_{2}$ and the CDW in $\mathrm{VSe}_{2}$ is quickly suppressed at small $x$. The screening of the Fe ions will be more effective (i. e. the local induced CDW will have a smaller amplitude and a smaller range). Thus the size of the random potential will be smaller in $\mathrm{VSe}_{2}$.

Both reasons make the occurrence of Anderson localization less tikely.

5. Conclusions. $-\mathrm{V}_{1-x} \mathrm{Fe}_{x} \mathrm{Se}_{2}$ has the $\mathrm{CdI}_{2}$ (1T) structure for $x \leqslant \frac{1}{2}$. Since the Fe appears to be divalent (low spin), at large $x$ the formal selenium valence must be less than $2^{-}$and the $V$ will be $5^{+}$. This is not unexpected, since the $\mathrm{V}$ d-band and Se p-band overlap, and at $x=\frac{1}{2}$ we expect 0.5 holes per Se.

The CDW state in $\mathrm{VSe}_{2}$ is eliminated by a small amount of cation substitution $(\approx 3 \%$ ). In contrast to $1 \mathrm{~T}-\mathrm{Ta}_{1-x} \mathrm{Fe}_{x} \mathrm{Se}_{2}$, no low spin-high spin transition is observed in $1 \mathrm{~T}-\mathrm{V}-\mathrm{Fe}_{x} \mathrm{Se}_{2}$. We have not observed an increasing resistivity at low temperatures expected from a possible Anderson localization, but the measurements are yet to be extended to higher Fe concentrations than $x=0.2$.

Acknowledgements. - Our understanding of the physical phenomena in layered compounds is the result of collaboration with many others including : J. D. Axe, M. Eibschutz, S. Mahajan, D. E. Moncton and J. A. Wilson and discussions with M. E. Lines and T. M. Rice. We thank A. H. Thompson for discussing his results on $\mathrm{VSe}_{2}$ prior to their publication.

\section{References}

[1] Wilson, J. A., Di Salvo, F. J. and Mahajan, S., $A d v$. Phys. 24 (1975) 117.

[2] Moncton, D. E., Axe, J. D., and Di Salvo, F. J., Phys. Rev. Lett. 34 (1975) 734.

[3] Di Salvo, F. J., Wilson, J. A., Bagley, B. G. and WaszCZAK, J. V., Phys. Rev. B 12 (1975) 2220.

[4] McMillan, W. L., Phys. Rev. B 12 (1975) 1187.

[5] Thompson, A. H., Phys. Rev. Lett. 34, (1975) 520, and private communication.

[6] Williams, P. M., Physics and Chemistry of Materials with Layered Structures, Vol. 2, pub. Reidel Holland, 1976

[7] Di Salvo, F. J., Moncton, D. E., Wilson, J. A. and WASzCZAK, J. V., Bull. Am. Phys. Soc. 21 (1976) 261, and to be published.

[8] Mattheiss, L. F., Phys. Rev. B 8 (1973) 3719.

[9] Rice, T. M. and ScotT, G. K., Phys. Rev. Lett. 35 (1975) 120.

[10] The estimates from reflectivity and heat capacity measurements give much smaller numbers than those obtained here from the change in susceptibility. See for example : Barker, A. S., Jr., Ditzenderger, J. A. and Di Salyo, F, J., Phys. Rev. B 12, (1975) 2049.

[11] Di Salvo, F. J., Wilson, J. A. and Waszczak, J. V. Phys. Rev. Lett. 36 (1976) 885.

[12] Eibschutz, M. and Di Salvo, F. J., Phys, Rev. Lett. 36 (1976) 104.

[13] Mott, N. F., Pepper, M., Pollett, S., Wallis, R. H. and Adkins, C. J., Proc. R. Soc. A 345 (1975) 169.

[14] Wertheim, G. K., Di Salvo, F. J. and Chiang, S., Phys. Lett. 54A (1975) 304.

[15] Hughes, H. B. and Pollak, R. A., Comm. Phys. 1 (1976) 61
[16] Shepherd, F. R. and Williams, P. M., J: Phys. C. 7 (1974) 4427.

[17] We note that isoelectronic $\mathrm{LiTiSe}_{2}$ has a large Pauli paramagnetic susceptibility and a decrease at low temperatures similar to $\mathrm{VSe}_{2}$, see MURPHY, D. W, DI SALvo, F. J., Hull, G. W., Jr. and Waszczak, J. V., Inorg. Chem. 15 (1976) 17.

[18] In all layered compounds studied to date $\mathrm{Fe}$ has a magnetic moment when intercalated, see for example: ErBsChutz, M., Di Salvo, F. J., Hull, G. W., Jr. and Mahajan, S., Appl. Phys. Let. 27 (1975) 464. The intercalated $\mathrm{V}$ in $\mathrm{V}_{1+x} \mathrm{Se}_{2}$ is also known to have a magnetic moment, see for example : SilberNAGEL, B. G., Thompson, A. H. and Gamble, F. R., 20th Anmual Conf. on Magnetism and Magnetic Materials, AIP Conf. Proceedings 24 (1975) 380.

[19] In Landau models the free energy difference between a CDW state with amplitude $\psi$ and the normal state is approximately (to lowest order) $\Delta F=F_{\mathrm{CDW}}-F_{\text {normal }}$ $\alpha a^{\prime}\left(T-T_{0}\right) \psi^{2}$. McMillan showed (Ref. [4]) that an impurity will induce a large local $\psi_{\mathrm{imp}}$ even above $T_{0}$ where $\Delta F>0$, with $\psi_{\text {imp }} \alpha\left(a^{\prime}\left|T-T_{0}\right|\right)^{-1 / 4}$ (for $T>T_{0}$ ). When impurities suppress the CDW state, i. e. $\Delta F>0$ at $T=0$, local distortions about each impurity are still expected, but this state is equilvalent to having a negative $T_{0}$. If the $\mathrm{CDW}$ is incipient, i. e. $\Delta F(T=0)>0$ but small, then $\left|T_{0}\right|$ will be small and the local distortions will still be quite large. These distortions produce diffuse scattering which peaks at the CDW wave vector $q$, even when the CDW is not the uniform ground state of the system at $T=0$. 